\title{
SYMPOSIUM
}

\section{General Symposium}

A. STERNS (Convenor). Three new approaches to medication adherence to ensure a better self. Gerontechnology 2018;17(Suppl):48s; https://doi.org/10.4017/gt.2018.17.s.048.00 Participants A. STERNS (USA), H. KORT (NETHERLANDS), K. CROSSLAND (USA), H. STERNS, Discussant (USA). Issue Medication nonadherence is a leading cause of loss of independence and leads to costly emergency services, hospitalizations, and death. Practitioners have advanced beyond digital alarms and 7-day organizers to create behavior focused cognitive prosthetic technologies. Content This symposium presents three different approaches with different underlying theoretical perspectives but with the identical goals of high adherence and better health outcomes. The A. Sterns presentation will review a National Institute of Health funded smartphone-based platform for empowering patients and healthcare professionals to manage medications together. The platform consists of a cloud-based management center, an app, and a unique Internet-of-Things (loT) pill dispensing device. The Kort presentation will present a review of the MedGuide project funded by the EUs Ambient Assistance Living (AAL) program. The aim of the MedGUIDE project is to develop a tool which gives: (1) A deeper understanding of the daily activities of persons with dementia (PwD); (2) Provides insight into the actual medication use, side effects, and adherence; and (3) A tool to support medication adherence. In the symposium a focus is laid on the users' needs and requirements for this tool. The Crosland presentation will describe an innovative technology that combines behavioral technology with automation using an inexpensive standard pill box with machine readable markers (Quick Response codes) that are scanned by a smart phone application. Structure The presentation will consist of three 10 to 15 -minute presentations that each includes an explanation of the theoretical background of the medication adherence approach, a review and demonstration of the components of the system, and research most recently completed using the technological approaches. The presentations will be followed by a discussion of the papers by Dr. Harvey Sterns, a past president of Division 20 of the American Psychological Association (APA) and the Association for Gerontology in Higher Education (AGHE) and a recipient of numerous life-time achievement awards in the field of gerontology. The discussion will begin with comments from Dr. Sterns and then be opened up for questions from the audience. Conclusion Attendees will be rewarded with a review of three theoretical approaches to medication adherence, presentation of each technology, and examples of using the technological approaches.

\section{References}

1. Chan, M., Nicklason, F., \& Vial, J.H. (2001). Adverse drug events as a cause of hospital admission in the elderly. Internal Medicine Journal; 31 (4):199-205

2. Sterns, A. A. \& Riley, T. (2016). Improving health literacy and health outcomes using cognitive prosthetic devices. In K. Kopera-Frye (Ed.) Health Literacy Among Older Adults. New York: Springer

Keywords: cognitive impairment, medication adherence, mHealth, eHealth Address: Management and Information Systems, Kent State University, Kent, OH: iRxReminder LLC, 1768 East $25^{\text {th }}$ Street, Suite $\# 308$, Cleveland $\mathrm{OH} 44114$;

E: asterns@kent.edu 


\section{SYMPOSIUM}

\section{General Symposium}

A. STERNS, J. SMITH, J. HUGHES. Smart home technologies as cognitive prosthetics to extend older adult independence. Gerontechnology 2018;17(Suppl):49s; https://doi.org/10.4017/gt.2018.17.s.049.00 Purpose Not properly taking medications results in 700,000 emergency room visits, 341,000 hospitalizations, and 125,000 deaths annually ${ }^{1}$. Patients have been, for the most part, on their own when it comes to making sure medications are taken correctly once prescribed. They have had a few popular tools including the 7-day pillbox organizer and the digital watch. These have proven inadequate as the number of ER visits, hospitalizations, and deaths attest. A new group of technologies has emerged to support improving medication taking success. Because these technologies are designed to help overcome behavioral and cognitive challenges of medication taking we refer to them as cognitive prosthetics ${ }^{2}$. The success of these prosthetics requires understanding the underlying behaviors and cognitive challenges. Method We describe an organizing model to better understand the ability of these technologies to deliver care in the home and independent living settings bridging care and for use in self-management of chronic conditions. Our organizing model focuses on the degree of support and the degree of integration with the healthcare professional. We have developed and successfully tested a technology platform called iRxReminder, created for supporting patient medication adherence. iRxReminder's platform consists of three components: (1) a cloud-based iRxControl center, (2) smartphone apps, and (3) an Internet-of-Things (IoT) medication dispensing device (Figure 1). Symptoms, side effects, and other health information are tracked in parallel using interoperating smartphone apps. This platform is ideal for integration with healthcare-focused smart homes. The technology extends to utilize cues such as lighting (changing a bulb from white to green) as well as smart device alerting. The platform empowers patients through the use of a dosing window. When the dosing window opens, the LED on the pod turns green, and the medication may be taken. In the middle of the dosing window plus 5-minutes the alerts are sent. The alert is always meaningful. We call this approach smart alerting. Results \& Discussion We present two studies that have been completed demonstrating significantly improved medication adherence to over $80 \%$ with individuals recovering $(\mathrm{Xage}=65)$ from transient ischemic attacks (TIAs or mini-strokes) and patients living with heart failure $(X a g e=72)$. We share two difficult home health challenges, managing complex medication schedules and wandering can be solved with technology supported medication reminding and access control technologies utilized in a home setting.

\section{References}

1. Viswanathan M, Golin CE, Jones CD, Ashok M, Blalock SJ, Wines RCM, Coker-Schwimmer EJL, Rosen DL, Sista P, Lohr KN. Interventions to Improve Adherence to Self-administered Medications for Chronic Diseases in the United States: A Systematic Review. Ann Intern Med. 2012;157:785-795; https://doi.org/10.7326/00034819-157-11-201212040-00538

2. Sterns AA, Mayhorn CB. Persuasive pillboxes: Improving medication adherence with personal digital assistants. In de Kort Y, Jsselsteijn JI (Eds.), Persuasive technologies. New York: Springer. 2006

3. Sterns AA. App development in the context of smart homes for healthcare. In (Ed.) van Hoof J, Demiris G, Wouters EJ. Handbook of Smart Homes, Health Care and Well-Being. New York: Springer. 2016

Keywords: medication adherence, cognitive prosthetic, mHealth, eHealth

Address: iRxReminder, 1768 East $25^{\text {th }}$ Street, Suite $\# 308$, Cleveland, $\mathrm{OH} 44114$, USA;

E: asterns@kent.edu

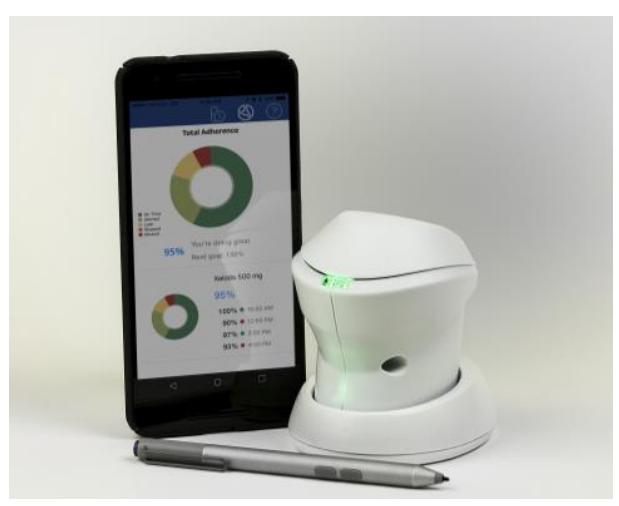

Figure 1. The iLidRx pill dispensing pod and the iRxCapture app interoperate to empower patients to achieve high adherence and better 


\section{SYMPOSIUM}

\section{General Symposium}

H.S.M. KORT. MedGUIDE: A review of the EU initiative on improving medication adherence for persons living with dementia. Gerontechnology 2018;17(Suppl):50s; https://doi.org/10.4017/gt.2018.17.s.050.00 Purpose The MedGUIDE project is a project under the Ambient Assistance Living (AAL) Funding scheme of the EU ${ }^{1}$. In this project, researchers, designers, practitioners, people with early stage dementia, and their carers are working together to design a tool for medication adherence. Dementia is a long-term condition leading to deterioration of cognitive abilities and limitations in daily activities which restrict people to live independently. It is estimated that world-wide almost 36 million people have dementia. The dementia syndrome usually occurs in combination with other chronic disorders. People with dementia (PwD) have to take several medications to control their symptoms. The symptoms in the dementia syndrome such as loss of time, orientation and memory, daily challenges the PWD in their medication adherence. Method The aim of the MedGUIDE project is to develop a tool which gives: (1) A deeper understanding of the daily activities of PwD; (2) Provides insight in the actual medication use, side effects and adherence; (3) A tool to support medication adherence. In the symposium a focus is laid on the users' needs and requirements for this tool. Results \& Discussion In the symposium, a focus is laid on the users' needs and requirements for this tool. Over $60 \%$ of people with dementia in Europe have trouble with over- and under-treatment of dementia-related medications, the target market of MedGUIDE is significant ${ }^{2}$. The MedGUIDE business model targets professional care providers (e.g.: pharmacy networks), who can use MedGUIDE to improve medication management and thereby better support their elderly patients. Furthermore, the consortium aims to also directly target health insurers, since the highlevel efficiency gains can only be incentivized on a meta-level.

\section{References}

1. Stefanov DH, Bien Z, Bang WC. The smart house for older persons and persons with physical disabilities: Structure, technology arrangements, and perspectives. IEEE Transactions on Neural Systems and Rehabilitation Engineering. 2004;12(2):228-250

2. Brewer L, Bennett K, McGreevy C. Eur J Clin Pharmacol 2013;69:1467; https://doi.org/10.1007/s00228-0131483-y

Keywords: medication adherence, assisted Living, eHealth Address: Bolognalaan 101, 3584 CJ Utrecht, The Netherlands; E: Helianthe.Kort@hu.nl 


\section{SYMPOSIUM}

\section{General Symposium}

K.A. CROSLAND, J. MOORE, W.D. KEARNS. An innovative inexpensive behaviourally-based medication management application. Gerontechnology 2018;17(Suppl):51s; https://doi.org/10.4017/gt.2018.17.s.051.00 Purpose As many as $26 \%$ of hospital admissions have been revealed to be due to medication noncompliance and omission of medication or cessation of indicated treatment, and $53.4 \%$ of these instances were found to be preventable ${ }^{1}$. Advances in automation have led to smart medication boxes to prompt and record medication compliance in the home. However, these devices can be very expensive, have complex interfaces challenging even normal elders and vex those with diminished cognitive capacity due to their medical conditions. In addition, these devices only signal when medications should be taken, but deliver no consequences to sustain motivation. This design oversight generates compliance rates hovering at 60 to $70 \%$ even with automated prompting - rates that should be much higher based upon behavioral research on medication compliance that has shown that repeatedly delivering positive reinforcers contingent, on medication-taking behaviors significantly increases compliance in normal adults/elderly ${ }^{2,3,4}$. Method This presentation will describe an innovative technology that combines behavioral technology with automation using an inexpensive standard pill box with machine readable markers (Quick Response codes) that are scanned by a smart phone application. The smart phone application records time and date information when the medication is removed from the pill box to determine if mediations are consumed according to the prescribed schedule. The application also employs behavioral technology that provides prompts to take medication, motivational information to the user, and positive reinforcement contingencies for medication consumption. Users can earn monetary rewards, token or point rewards to later exchange for monetary rewards, or other items found to be preferred. The reinforcement schedule can be adjusted to schedules found to sustain behavior over long periods of time, such as variable schedules of reinforcement. This presentation will describe the technology and present some preliminary pilot testing data. Results \& Discussion This type of technology could enhance independent living skills and be disseminated to a wide range of users including elderly, individuals with traumatic brain injury, and others with mild or chronic medical conditions where medication compliance is essential.

\section{References}

1. Chan M, Nicklason F, Vial JH. Adverse drug events as a cause of hospital admission in the elderly. Internal Medicine Journal; 2001;31(4):199-205

2. Dapcich-Miura E, Hovell, MF. Contingency management of adherence to a complex medical regimen in an elderly heart patient. Behavior Therapy: 1979;10(2):193-201

3. Petry NM, Alessi SM, Bryne S, White WB. Reinforcing adherence to antihypertensive medications. Journal of Clinical Hypertension. 2015;17(1):33-38

4. Raiff BR, Jarvis BP, Dallery J. Text-message reminders plus incentives increase adherence to antidiabetic medication in adults with type 2 diabetes. Journal of Applied Behavior Analysis. 2016;49(4): 947-953

Keywords: medication adherence, behavior analysis, smart phone application, reinforcement Address: Child \& Family Studies, University of South Florida, Tampa, Florida, USA;

E: Crosland@usf.edu 